\title{
Processing, Inversion and Imaging of Seismic Marine Data of Basin of Jequitinhonha
}

Wildney W. S. Vieira, Lourenildo W. B. Leite, Raimundo N. C. Carneiro and Melquezedeque P. Aquino, UFPA, Brazil

Copyright 2019, SBGf - Sociedade Brasileira de Geofísica.

This paper was prepared for presentation at the $16^{\text {th }}$ International Congress of the Brazilian Geophysical Society, held in Rio de Janeiro, Brazil, 19-22 August, 2019.

Contents of this paper were reviewed by the Technical Committee of the $16^{\text {th }}$ International Congress of The Brazilian Geophysical Society and do not necessarily represent any position of the SBGf, its officers or members. Electronic reproduction or storage of any part of this paper for commercial purposes without the written consent of The Brazilian Geophysical Society is prohibited.

\section{Abstract}

This work is focused on processing, and imaging of the selected L2140266 line of the Jequitinhonha Basin, Bahia, based on the NMO and CRS stacking methodologies and the Inversion of the NIP wave, where the of post-stacking Kirchhoff migration in time is in depth, and common to all techniques. Beyond in addition, the partial-CRS stack was applied to densification of CMPs. Several tests were performed to optimize parameters, operator openings, minimize the objective function and regularize the parameters of the model, and as a result the flowchart of generated better visual quality of the sections, the main results compared in the course of the work for both methodologies.

\section{INTRODUCION}

The exploitation of marine energy resources requires more and more high technology for success and optimization of costs. In this direction, the academy and projects with economic resources directed to develop research and contribute to the formation of Human resources for oil and gas. Being thus, the area of geophysical exploration, particularly the seismic, has fundamental importance in obtaining images of terrestrial subsurface in basin areas sedimentary potential and risk reduction exploratory.

The use of the three methodologies used has the purpose of complementing and increasing the information on the characteristics of the subsurface, and give more options for geological interpretations of the results obtained. The main results are stacked and migrated sections discussed in more detail detailed in the sequence of the work.

For the processing, inversion and imaging the applications were used CWP/Un*x Forel et al. (2005), crsstack Mann (2003), crsstack_supergathers Baykulov (2008) and niptomo Duveneck (2004).

\section{METHODOLOGY / RESEARCH PROBLEM}

The NMO stacking methodology is based on the model of flat-horizontal layers, whose time of transition to primary reflections is given by a hyperbolic approximation given by Yilmaz (1988) :

$$
t^{2}(x)=t^{2}(0)+\frac{x^{2}}{v_{N M O}^{2}},
$$

where $x$ is the source-receiver spacing, $t(x)$ the time transit, $t(0)$ the double-time simple vertical and $v_{N M O}$ the normal overtemperature speed. It is necessary to A model of velocity distribution obtained through of paired marking $\left(v_{N M O}, t_{0}\right)$ on the coherence map Semblance, and that serves the correction, stacking and migration.

The CRS stack, initially described by MÃ $\frac{1}{4}$ ller (1999) and Mann (2002), is based on the layers bounded by curved interfaces, and considered independent of the speed model, where the transit for primary reflections is given by the approximation hyperbolic:

$$
\begin{gathered}
t_{C R S}^{2}\left(x_{m}, h\right)=\left[t(0)+2 \frac{\sin \beta_{0}}{v_{0}}\left(x_{m}-x_{0}\right)\right]^{2} \\
+2 t(0) \frac{\cos ^{2} \beta_{0}}{v_{0}}\left[\frac{\left(x_{m}-x_{0}\right)^{2}}{R_{N}}+\frac{h^{2}}{R_{N I P}}\right]^{2},
\end{gathered}
$$

being $x_{m}$ the common-mid-point, $v_{0}$ the next speed to the point of emergency and $h$ the source-receiver half-distance. In this method we retrieve the trio of attributes of the two hypothetical waves $N$ and NIP through the solution of a non-linear triparet optimization problem, and Which are: the emergency angle $\beta_{0}$, the radius of curvature $R_{N}$ and the radius of curvature $R_{N I P}$. These attributes carry Information on slope, depth and shape of the reflecting interfaces. In order to improve These results we opted for using the process Of CRS-partial stacking described by Baykulov \& Gajewski (2008), and that produced sections of better reason signal/noise ratio. This process consists of the interpolation of CMP families to the data cube with the objective of Densify the mesh prior to stacking CRS under The condition of super-families.

The tomographic inversion of the wave NIP, developed by Duveneck (2004),

aims at establishing a distributing speeds with lateral variation, to serve in-depth imaging, and uses the CRS attributes through the data marked in section ZO stacked. The problem of non-linear curve fitting in the of the minimum squares between the markings of the parameters of the input data $\mathbf{d},\left[\tau_{0}, M_{N I P}^{(\xi)}, p^{(\xi)}, \xi_{0}\right]$, and the corresponding quantities of the direct model $\mathbf{m},[x, z, \theta]^{(N I P)}$, ssociated with the PIN point of the model. Such mismatch and minimized by the cost function in the form:

$$
\begin{gathered}
S(\mathbf{m})=\frac{1}{2} \Delta \mathbf{d}^{T}(\mathbf{m}) \mathbf{C}_{D}^{-1} \Delta \mathbf{d}(\mathbf{m})+\frac{1}{2} \varepsilon^{\prime \prime} \mathbf{m}^{(v) T} \mathbf{D}^{\prime \prime} \mathbf{m}^{(v)} \\
+\frac{1}{2} \varepsilon^{\prime \prime} \mathbf{m}^{(v) T} \mathbf{D}^{\prime \prime} \mathbf{m}^{(v)}
\end{gathered}
$$

where $\Delta \mathbf{d}(\mathbf{m})=\mathbf{d}-\mathbf{f}(\mathbf{m})$ is the vector deviation of the data, $\mathbf{C}_{D}$ the covariance matrix of the data, $\mathbf{D}^{\prime \prime}$ the regularization and $\varepsilon^{\prime \prime}$ the weighting factor between the parties regularization of the inversion operator. 


\section{RESULTS}

The parameters and operator openings chosen for the processing of this line, as well as the order of the in the flowcharts were obtained by means of Of exhaustive tests, and the most satisfactory results in the sense of higher signal-to-noise ratio has its main results presented in the sequence.

The pre-processing stage, performed by Lima (2010), was common to both methodologies, and included processes shown in Figure 1. For the insertion of the Geometry analysis of the field report, and the Information can be found in Table 1.

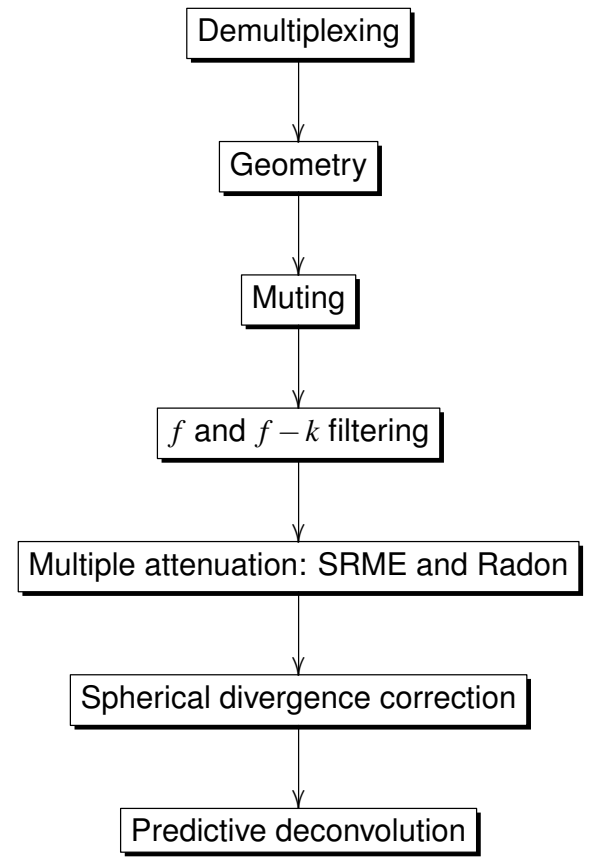

Figure 1: Preprocessing Flowchart.

\begin{tabular}{|c|c|}
\hline \multicolumn{2}{|c|}{ Acquisition Geometry Information } \\
\hline \hline Bowl & Jequitinhonha \\
\hline Line & L2140266 \\
\hline Year of acquisition & 1985 \\
\hline Configuration & Cable-drag \\
\hline Font Type & air-cannon \\
\hline Cable depth & $9 \mathrm{~m} \mathrm{a} 11 \mathrm{~m}$ \\
\hline Source depth & $9 \mathrm{~m}$ \\
\hline Cable length & $2975 \mathrm{~m}$ \\
\hline Shots number & 1589 \\
\hline receivers number & 120 \\
\hline shots interval & $25 \mathrm{~m}$ \\
\hline receivers interval & $25 \mathrm{~m}$ \\
\hline minimum offset & $150 \mathrm{~m}$ \\
\hline maximum offset & $3125 \mathrm{~m}$ \\
\hline Record time & $7.0 \mathrm{~s}$ \\
\hline Sampling interval & $4 \mathrm{~ms}$ \\
\hline
\end{tabular}

Table 1: Acquisition Geometry Information.

The exit data from this first stage, organized in the CMP family, served as input for the NMO and CRS. Thus, the NMO processing was and the steps are shown in Figure 2.

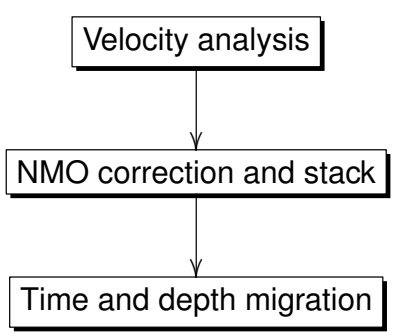

Figure 2: NMO stacking flowchart.

The speed models smoothed over time and in the depth, are shown in Figures 3 and 4, and were obtained, respectively, by markings in the semblance map and the conversion of average-quadratic velocities for intervals.

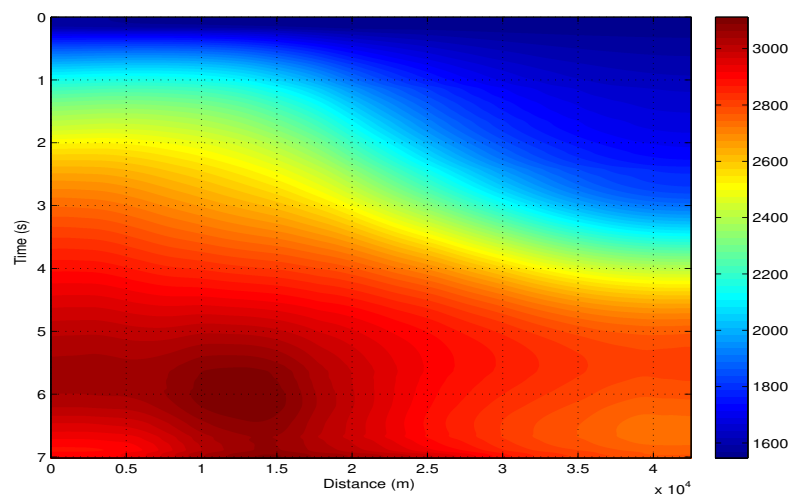

Figure 3: Speed model semblance in time.

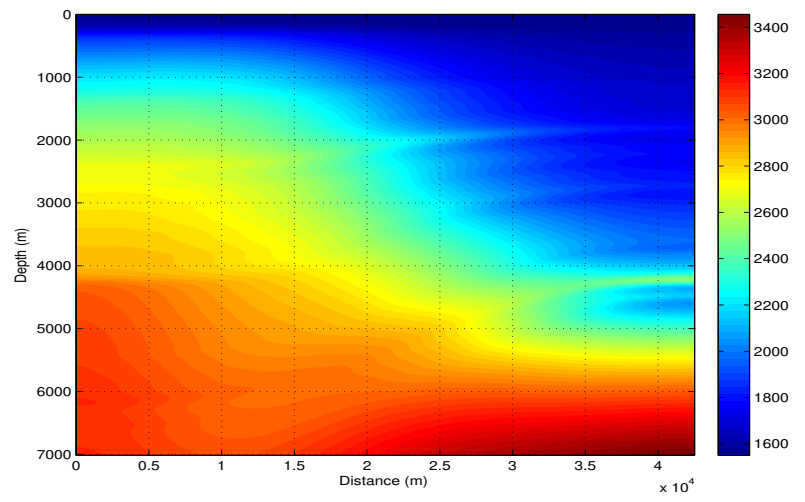

Figure 4: Semblance velocity model converted to depth.

After the NMO correction, the NMO given whose result is shown in Figura 5. 


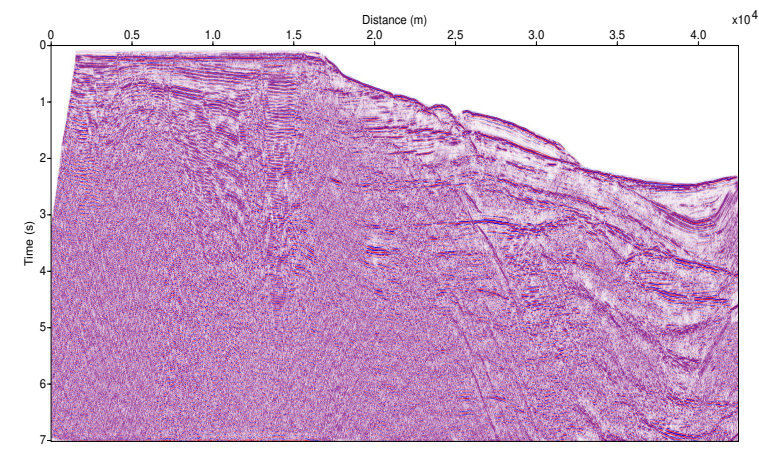

Figure 5: Stacked section NMO.

In this figure it is observed that most of the information recorded corresponds to shallow events and with good continuity. However, many diffractions as the depth increases. The results of post-stacking Kirchhoff migration, in time and depth, are shown in Figures 6 e 7 , respectively.

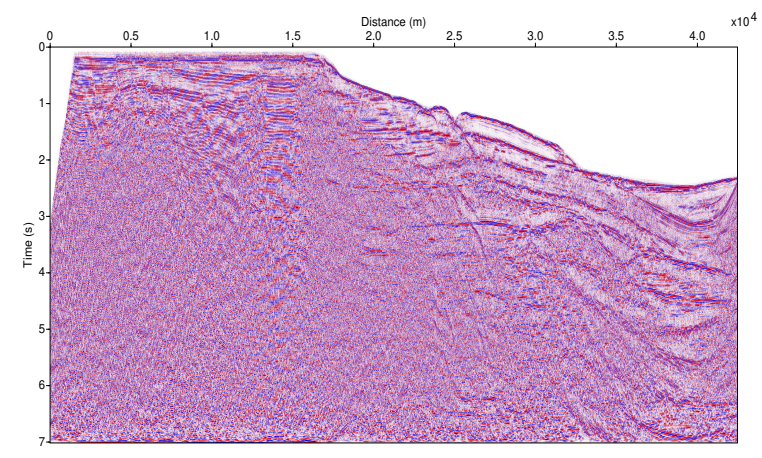

Figure 6: Kirchhoff migrated section in time.

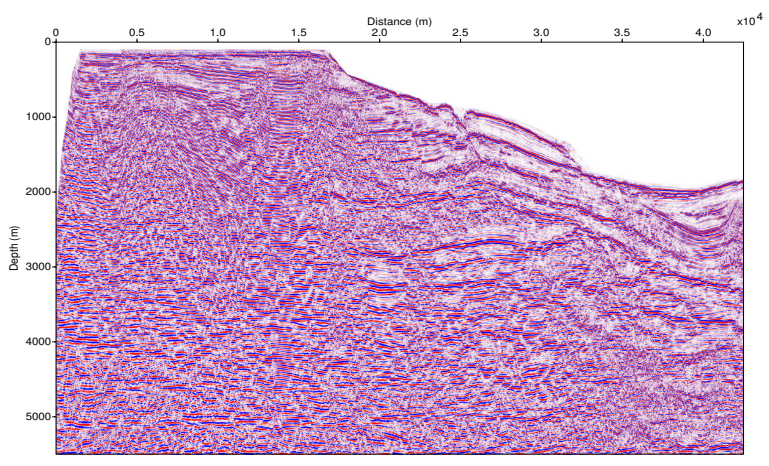

Figure 7: Section migrated Kirchhoff in depth.

Analyzing Figures 6 and 7 one can observe the small displacement of geological structures in relation to Figure 5 , the retrieval of the subhorizontalization of the structures, designed by the best continuity of reflectors, the collapse of diffractions and the correction of geological features. Section 7 is of superior quality than Figure 6 due to the recovery of events in large Depths and the absence of arcs on points diffractors ("smiles").
The process of densification of the data, called Partial CRS Stacking (see Figure 8), performed initially by Lima (2010) and and complemented by Silva (2012),

has the output data organized in super-families CMP, and that is input to the CRS stack, whose steps are shown in Figure 9.

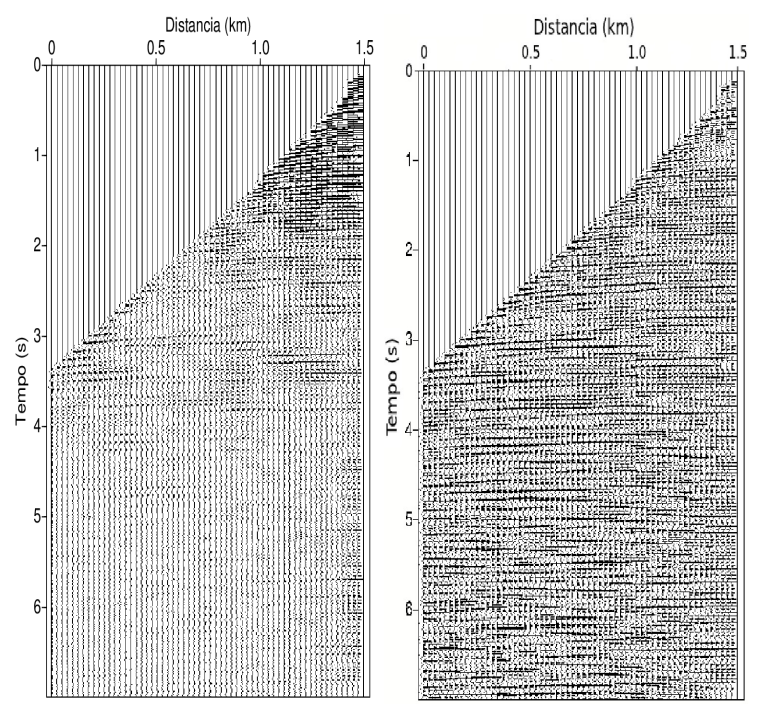

Figure 8: CMP 1010 family before and after densification of the pre-stacked die.

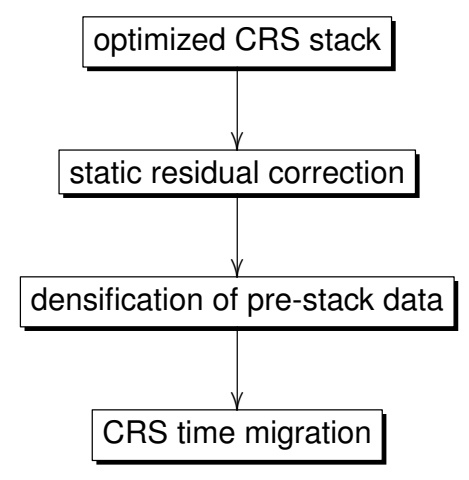

Figure 9: CRS stacking flowchart.

Following with the CRS stacking flowchart, Sub-products were selected, such as the CRS sections Stacked (see Figure 10) and migrated (see Figure 11) First Fresnel Zone Design. 


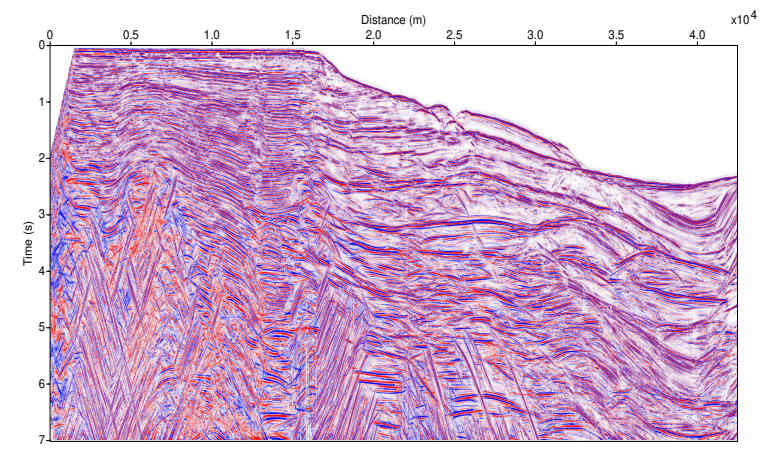

Figure 10: CRS stacked section.

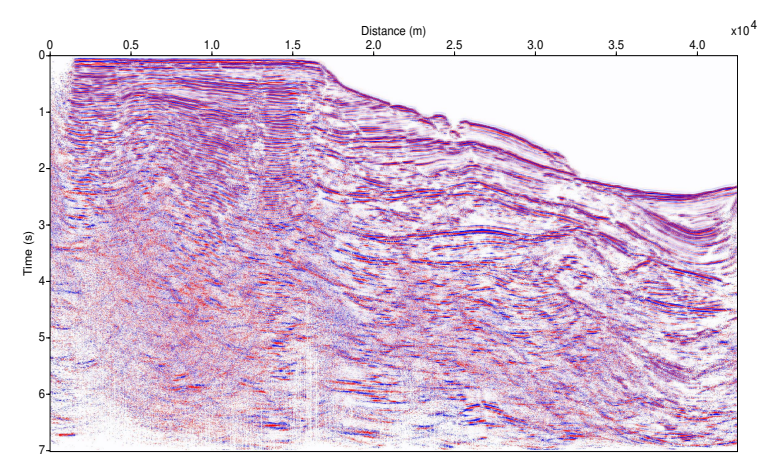

Figure 11: CRS-migrated section.

Figure 12 shows the Kirchhoff migration in time using the velocity model of Figure 3 and the section stacked CRS optimized of Figure 10.

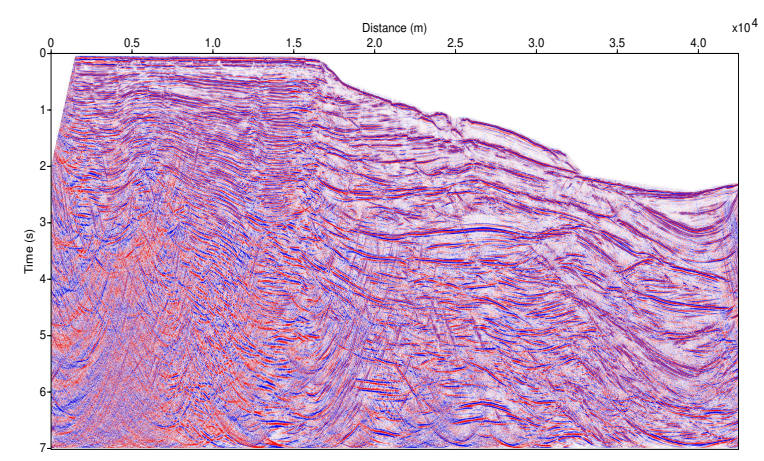

Figure 12: Kirchhoff migration using the speed semblance and stacking CRS.

The stacked section CRS presents better quality than the NMO in the sense of plus the structures due to better continuity of events Reflectors and the large amount of diffraction.

The enhancement of the free surface multiple is also larger, as the greater coherence for the reflection events primary.

On CRS migration, one can observe the collapse diffraction, the correction of geological faults, the Continuity of reflective events, the large amount of of granulations, the absence of arcs on the points Diffraction ("smiles") and the attenuation of multiple free surface.

The Kirchhoff migration in time using the speed semblance and the stacked CRS section shows many diffractions and a large amount of arcs above diffraction points ("smiles"), mainly for the events at great depths, demonstrating the low quality of the section and the incoherence of the methodology.

In the tomographic inversion of the NIP wave, whose shown in Figure 13, 2970 markings were made on the Domain $\left(\tau_{0}, \xi_{0}\right)$ about primary reflex events Of the stacked CRS section (see Figure 14).

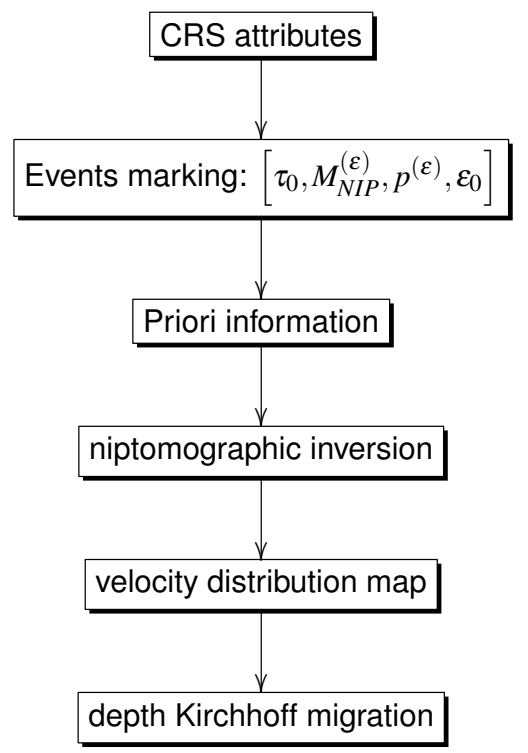

Figure 13: Flowchart of niptomographic inversion.

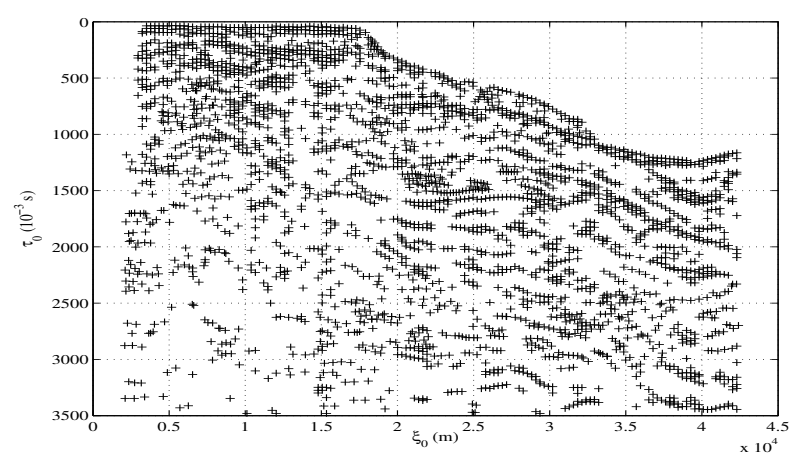

Figure 14: Map of the 2970 markings made on the section Stacked CRS.

Figure 15 shows the minimization of the cost function in Function of the number of iterations, where the Reduction of the mismatch value between the parameters of the Input and model data. 


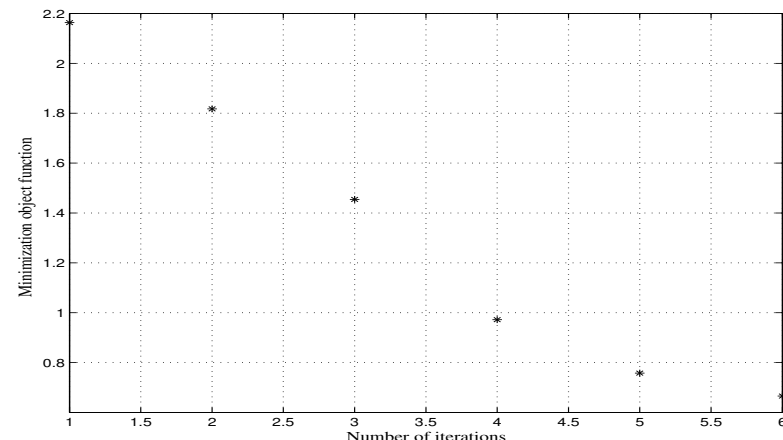

Figure 15: Minimizing the mismatch function according to the number of iterations.

The initial and final niptomographic velocity models, are shown, respectively, in Figures 16 e 17, where the great evolution of the final model is observed.

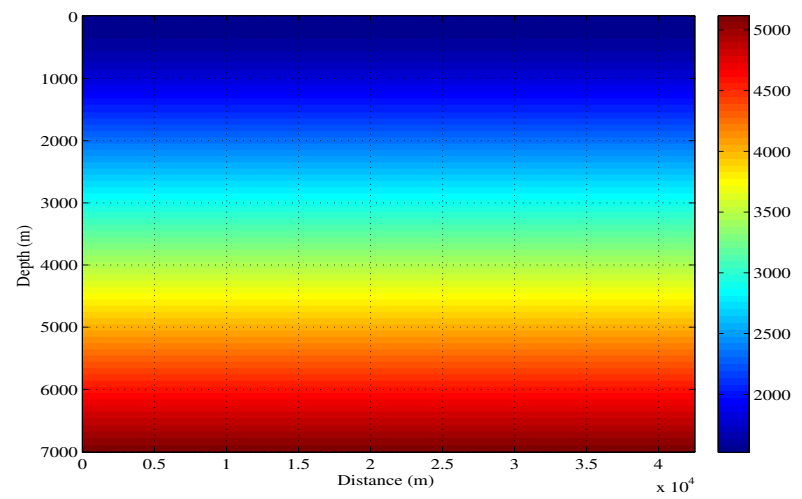

Figure 16: Initial niptomographic velocity model.

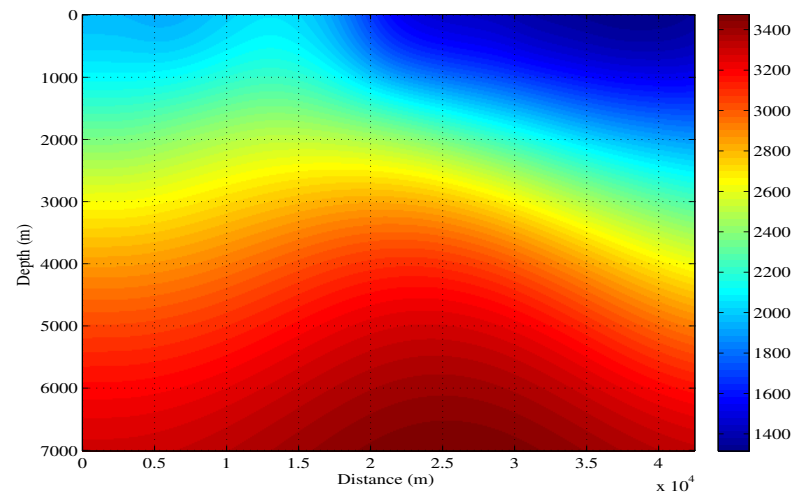

Figure 17: Final niptomographic velocity model.

Using the model of Figure 17, the Kirchhoff in depth, and the result is shown in Figure 18.

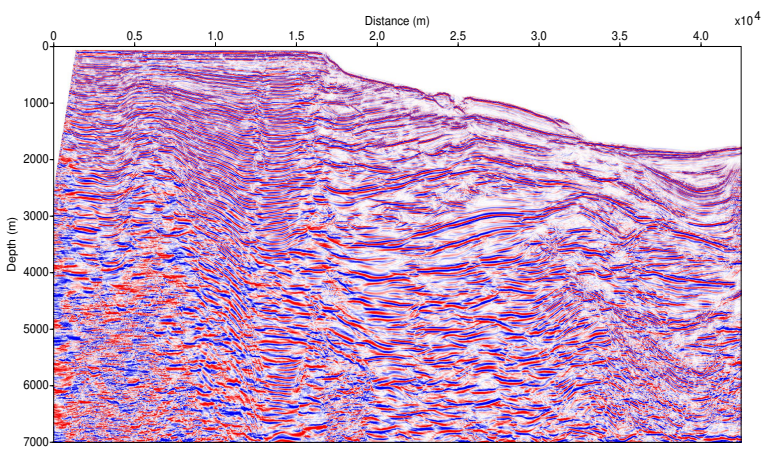

Figure 18: Section migrated Kirchhoff in depth.

The niptomographic velocity model, in addition to be similar to the speed model semblance (see Figure 4), shows with better definition the ocean floor, the distribution of the values of velocities in the deeper regions of the and contour lines with the different speed contrasts.

In the Kirchhoff migration the diffraction collapse in the deeper regions of the basin and the absence of above the diffractive points ("smiles").

\section{DISCUSSION AND CONCLUSIONS}

The conclusions on the results of the NMO and CRS, niptomographic inversion and migration Kirchhoff, are clearly showing difficulties and serious limitations, as well as cases that merit further. Attention to the implementation of the theories studied, and serve as a reference for future work.

From the comparative point of view between the sections obtained, the stacked CRS section has vertical resolution and horizontal section above the stacked NMO section, since in the CRS section one can better distinguish the interfaces and reflecting continuous structures through section, in addition to highlighting more clearly the of diffraction. The comparison between the migrated sections of these two methodologies shows that CRS migration Has better visual quality and continuity of events reflectors.

With regard to speed models it was possible to conclude that the obtained through niptomography has better visual quality than the speed model semblance, due to its better distribution of in the geological section, showing the coherence of methodology.

The comparison between the results of the Kirchhoff migration in depth shows that the section obtained using the niptomographic velocity model has quality superior to the respective section obtained through the velocity semblance, since there is great difference in the continuity of reflective events and in the collapse of diffraction.

As suggestions for future work, we propose the application of other post-depth based on equation such as PSPI (PhaseShift Plus Interpolation), SS (Split-Step), RTM (Reverse Time Migration), and FFD (Fourier Finite Difference), for comparison with the Kirchhoff migration, and the generalization of the results obtained with the CRS and the NIP wave tomography for the 3D case. 


\section{Acknowledgments}

The authors would like to thank the sponsorship of Project INCT-GP, and also in special to the Project PRH-06, that are present in part of this research work. We extend our thanks also to CAPES and CNPQ for the scholarships.

\section{References}

Baykulov, M., and Gajewski, D., 2008, Prestack seismic data enhancement with partial common reflection surface (crs) stack: Wave Inversion Technology, , no. 1, 57-73.

Baykulov, M., 2008, Partial crs stack user's manual: University of Hamburg.

Duveneck, E., 2004, Tomographic determination of seismic velocity models with kinematic wavefield attributes: University Karlsruhe.

Forel, D., Benz, T., and Pennington, W. D., 2005, Seismic data processing with seismic $u^{*} x$ - a $2 d$ seismic data processing primer:, volume 12 Society of Exploration Geophysicists.

Lima, H. M., 2010, Processamento e imageamento de dados sísmicos marinhos: Universidade Federal do Pará.

Mann, J., 2002, Extensions and applications of the common-reflection-surface stack method: Universitä Karlsruhe.

Mann, J., 2003, 2-d commom-reflection-surface stack user's manual: Universität Karlsruhe.

Müller, T., 1999, The commom reflection surface method - seismic imaging without explicit knowledge of the velocity model: Universitä Karlsruhe.

Silva, D. A. B., 2012, Processamento, inversão e imageamento de dados sísmicos marinhos: Universidade Federal do Pará.

Yilmaz, O., 1988, Seismic data processing:, volume 2 Society of Exploration Geophysicists. 